\title{
Bioengineering of Reporter Transgenes for Integrated Imaging with Magnetic Resonance, Fluorescence, and Electron Spectroscopy.
}

\section{Marek Malecki.}

\section{SABA University School of Medicine.}

For efficient monitoring of the new transgene constructs expression, it is necessary not only to monitor their life-time distribution within a whole body, but also their spatial distribution within tissues and cells as well as location within bio-macromolecular assemblies. This is accomplished through either labeling with antibodies tagged with reporter molecules or expressing fusion proteins containing reporting domains. Unfortunately, the imager allowing us to reveal the transgene products over such a wide spectrum of approaches does not exist. Hence, there is a strong need for correlations between various imaging modes [1]. Recently, we bioengineered the recombinant single-chain variable fragment ( $\mathrm{scFv}$ ) antibodies designed for simultaneous detection with magnetic resonance imaging (MRI) and positron emission tomography (PET) followed by molecular imaging with electron energy loss spectroscopy (EELS) [2]. In our previous work, we bioengineered the plasmid constructs driving organelle targeted expression of the transgene for a fusion protein with the integrated reporter molecules [3]. Here, we report the results of bioengineering of the constructs for transgenes, which products are detectable with MRI, fluorescence, and EELS.

The plasmid constructs were bioengineered into the previously described frame [2] in which the $\mathrm{scFv}$ site was replaced with the occludin coding sequence. The constructs were delivered into cultured human umbilical vein endothelial cells via receptor mediated gene transfer [3]. The cells were grown either as monolayers or within polystyrene sponges or as clusters injected into a nude mice. For preliminary evaluation of the transfection efficiency with fluorescence imaging, the cultured cell whole-mounts were suitable. For magnetic resonance imaging, the cell clusters and polystyrene sponges were appropriate. For electron energy loss spectroscopy, the cells from all strategies were rapidly frozen, freeze-substituted, embedded in LRW, and ultra-thin sectioned.

These newly bioengineered constructs open new avenues not only for studying dynamics of angiogenesis, but also endothelial cell response to oxidative stress or administration of pharmaceutics. These studies can now be pursued through monitoring of transgene expression via integrated imaging with magnetic resonance, fluorescence, and electron spectroscopy [4].

\section{References.}

[1] R. Albrecht et. al., Meth. Enzymol. 215, (1992) 456.

[2] M. Malecki et. al., Proc. Natl. Acad. Sci. U S A., 99 (2002) 213.

[3] M. Malecki et. al., Microsc. Microanal. 4 (Suppl. 2) (1998) 994.

[4] This research was supported by the NSF grants 9420056, 9522771, 9902020, and 0094016.

\section{Figure legends.}

The transgene constructs for fusion protein (occludin and integrated reporter molecule) were expressed in cultured human umbilical vein endothelial cells (HUVEC). The images were acquired with ultra-low-light, wide-field, computer deconvolution fluorescence microscopy. Horizontal field width $=50$ microns. 


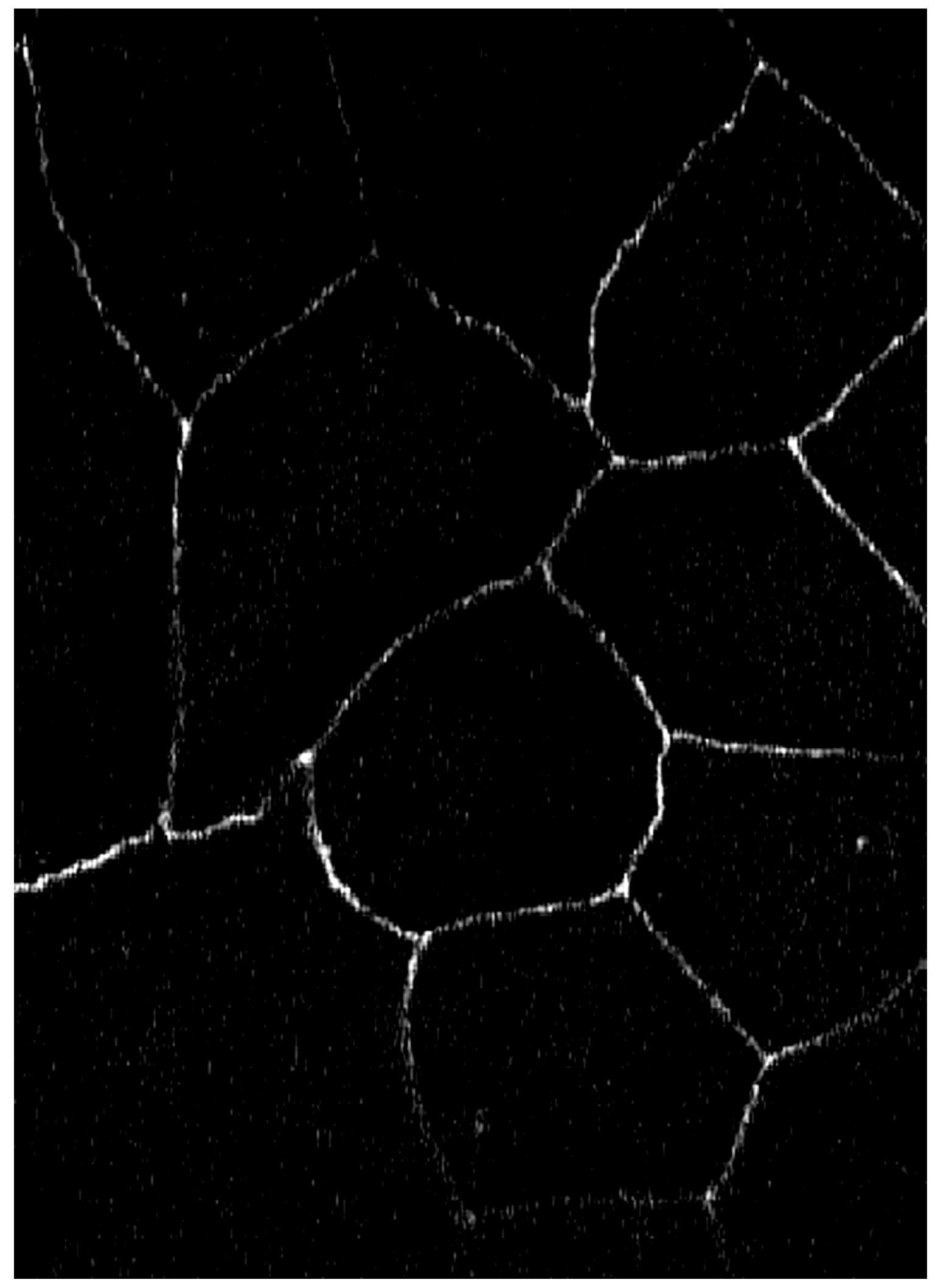

